\title{
Microbial-generated amyloids and Alzheimer's disease
} (AD)

\author{
James M. Hill ${ }^{1,2,3,4}$ and Walter J. Lukiw ${ }^{1,2,5 *}$ \\ ${ }^{1}$ Louisiana State University Neuroscience Center, Louisiana State University Health Sciences Center, New Orleans, LA, USA \\ ${ }^{2}$ Departments of Ophthalmology, Louisiana State University Health Sciences Center, New Orleans, LA, USA \\ ${ }_{3}^{3}$ Microbiology, Louisiana State University Health Sciences Center, New Orleans, LA, USA \\ ${ }^{4}$ Pharmacology, Louisiana State University Health Sciences Center, New Orleans, LA, USA \\ ${ }^{5}$ Neurology, Louisiana State University Health Sciences Center, New Orleans, LA, USA \\ *Correspondence: wlukiw@lsuhsc.edu
}

\section{Edited by:}

Myra Elizabeth Conway, The University of the West of England, UK

Reviewed by:

Daniel Ortuño-Sahagún, Centro Universitario de Ciencias de la Salud, Mexico

Vinood B. Patel, University of Westminster, UK

Jonathon Hull, University of the West of England, UK

Keywords: 42 amino acid amyloid-beta peptide (Aß42), Alzheimer's disease (AD), birefringence, central nervous system (CNS), Congo red, innate immunity, microbiome, toll receptor type 2 (TLR2)

\section{INTRODUCTION}

Atypical amyloid generation, folding, aggregation and impaired clearance are characteristic pathological features of human neurodegenerative disorders including Alzheimer's disease (AD). What is generally not appreciated is that a major secretory product of microbes is amyloid, and that the contribution of microbial amyloid to the pathophysiology of the human central nervous system (CNS) is potentially substantial. While earlier findings suggested that these amyloids may serve some immune-evasive strategy, it has recently become evident that humans have a tremendously heavy systemic burden of amyloid which may contribute to the pathology of progressive neurological diseases with an amyloidogenic component. This perspective will highlight some recent inroads made into our understanding of the enigmatic role that microbial amyloids may play in the homeostasis and pathology of the CNS with particular reference to $\mathrm{AD}$ wherever possible.

\section{AMYLOID: MICROBIAL AND CNS SOURCES}

"Amyloid" is a generic term for any aggregated, insoluble, lipoprotein-rich deposit exhibiting $\beta$-pleated sheet structures oriented perpendicular to the fibrillar axis (Steensma, 2001; Badtke et al., 2009; Blanco et al., 2012; Buxbaum and Linke, 2012). Amyloids are characterized by an apple-green birefringence $\left(\lambda_{\max } \sim 555 \mathrm{~nm}\right)$ when stained with the secondary diazo-dye Congo-red $\left(\lambda_{\max } \sim 498 \mathrm{~nm}\right)$ when viewed under polarized light (upon binding to amyloid, Congo Red displays bright fluorescence emission at $\lambda_{\max } \sim 614 \mathrm{~nm}$ after excitation at $\lambda_{\max } \sim 497 \mathrm{~nm}$; Alexandrov et al., 2011; O'Brien and Wong, 2011). Amyloid fibrillation is initiated by self-aggregation of protein monomersinto-dimers, oligomers and fibrils, which accumulate over time, and this process is thought to result from the hydrophobic nature of the aromatic amino-acid peptides comprising the primary sequence of the amyloid (O'Brien and Wong, 2011; Lukiw, 2012). The Congo red dye-based intercalation of $\beta$-pleated sheets, induction of a positive anisotropy that is polarized and directionally dependent, and generation of a measureable wavelength shift and apple-green birefringence is the hallmark of all amyloids and is the "gold standard" in the diagnosis of amyloidogenic disease (Linke, 2006; Buxbaum and Linke, 2012). The polymerization of amyloidogenic proteins is cooperative, and can be accelerated by amyloid aggregates derived from the same protein in a selective "seeding" process. The identification of the "amylome," a classification of amino acid sequences within proteins with internal, self-complementary interfaces and high fiber-forming propensity has improved our understanding of the capability of different proteins to form amyloids that contribute to "densedeposit" disease (Goldschmidt et al., 2010; O'Brien and Wong, 2011; Lukiw, 2012). The pathogenesis of diseases that accumulate amyloid, including $\mathrm{AD}$, all involve a marked inflammatory response at sites of amyloid deposition, and this is mediated by microglial cells, the "roving macrophages" of the CNS. Microglia appear to utilize molecular sensors on their external surface, such as the Toll-like receptor 2, TLR2, to recognize abnormal forms of amyloid and initiate a phagocytic or "clearance" response (Zhao et al., 2013; Ferrera et al., 2014; Jones et al., 2014). Here we describe a relatively recent collection of stimulating research at the crossroads of microbial and $\mathrm{AD}$ amyloids highlighting 5 recent, specific and illustrative insights into the potential contribution of microbial-derived amyloids to CNS amyloidogenesis and AD.

\section{MICROBIOME-DERIVED AMYLOIDS}

The microbiome is the aggregate of all microorganisms that reside on and within the human body, forming a complex ecosystem that includes the skin, oral and nasal mucosa, the urogenital and gastrointestinal (GI) tracts. The microbiome of the GI tract is by far the largest reservoir of microbes in the human body, containing about $10^{14}$ microbes; over 99\% of microbiota in the GI tract are anaerobic bacteria, with fungi, protozoa, archaebacteria and other microorganisms 
making up the remainder (Bhattacharjee and Lukiw, 2013; Hill et al., 2014; Lin et al., 2014). Prokaryotic cells of the human microbiome outnumber human eukaryotic host cells by about 100 to 1 , and collectively microbial genes outnumber host genes by about 150 to 1 (Hill et al., 2014; Lin et al., 2014). Recent microbiome analysis has revealed that the majority (98\%) of all GI tract species belongs to only 4 major bacterial divisions: the gram-positive Firmicutes (64\%) and Actinobacteria (3\%) and the gram-negative Bacteroidetes (23\%) and Proteobacteria (8\%). The remaining 2\% consist of minor taxonomic divisions which are quite diverse (Hattori and Taylor, 2009; Bhattacharjee and Lukiw, 2013; Schwartz and Boles, 2013). Many different microbiome species including fungi and bacteria secrete amyloid (Hill et al., 2014; Syed and Boles, 2014). For example, amyloids are associated with fungal surface-structures and the recent observation of amyloidogenic fungal proteins and diffuse mycoses in the blood of $\mathrm{AD}$ patients suggest that chronic fungal infection increases $\mathrm{AD}$ risk (Alonso et al., 2014a,b; Hill et al., 2014). To cite one other recent example, in Escherichia coli extracellular amyloids known as curli fibers, composed of the major structural subunit CsgA, are a common secretory component that facilitate surface attachment and adhesion, biofilm development and protection against host defenses (Schwartz and Boles, 2013; Asti and Gioglio, 2014). Biofilms represent a matrix of extracellular polymeric amyloids and other lipoproteins in various structural forms. Interestingly, the extracellular $17.7 \mathrm{kDa}$ CsgA amyloid precursor contains a pathogen-associated molecular pattern (PAMP) that, like the $\mathrm{A} \beta 42$ peptide, is recognized by the human immune system TLR2 (Zhou et al., 2012). An expanding list of bacterial amyloid systems include those associated with gramnegative species of Streptomyces, Bacillus, Pseudomonas, Staphylococcus and others, suggesting that functional amyloids are a widespread phenomenon utilized by a wide diversity of microbiome-bacteria (Schwartz and Boles, 2013; Asti and Gioglio, 2014; Hill et al., 2014). Indeed the extremely large number and variety of microbiome bacteria and their capability to produce vast quantities of amyloids indicates that human physiology may be potentially exposed to a tremendous systemic amyloid burden, especially during aging when the GI tract epithelial and blood-brain barriers become significantly more restructured and permeable (Bhattacharjee and Lukiw, 2013; Marques et al., 2013; Oakley and Tharakan, 2014).

\section{THE AMYLOID PEPTIDES OF AD AND ENDOTOXIN-MEDIATED INFLAMMATION}

A $\sim 770$ amino acid type 3 transmembrane $\beta$-amyloid precursor protein ( $\beta$ APP), through interaction with membrane proteins and tandem secretase cleavage yields a series of ragged $\mathrm{A} \beta$ peptide monomers, two of the most abundant being the 40 and 42 amino acid peptides $A \beta 40$ and A 342 (Van Broeck et al., 2007; O'Brien and Wong, 2011; Zhang et al., 2012). $\mathrm{A} \beta 40$ peptides associate with the endothelium of the cerebral vasculature, and the more neurotoxic, albeit less abundant, hydrophobic $A \beta 42$ species form the nuclei of the senile plaque (SP) lesions of AD (Alexandrov et al., 2011; O'Brien and Wong, 2011). Interestingly, the extra two hydrophobic amino acids in $A \beta 42$ (vs. A $\beta 40$ ) appear to convey many of the toxic biophysical attributes and selfaggregation of this slightly larger molecule (Zhou et al., 2011; Teng et al., 2012; Zhang et al., 2012). The recognition of A $\beta 42$ peptides and their misfolded aggregates by microglial-surveillance systems, and the inability of microglial cells to deal with these toxic, pro-inflammatory inclusions are thought to form the molecular basis for the elevated oxidative stress, aberrant immune-activation and chronic inflammation characteristic of AD brain (Armstrong, 2006; Cui et al., 2007; Van Broeck et al., 2007; Boutajangout and Wisniewski, 2013; Furukawa and Nukina, 2013; Ferrera et al., 2014; Serpente et al., 2014; Takeda et al., 2014). Interestingly (i) $\beta$ APP-derived $A \beta 42$ peptides induce a pattern of expression of inflammatory genes typical of the classical immune- and inflammatoryresponse induced by infectious agents such as bacterial lipopolysaccharide (LPS), a common endotoxin of the outer membrane of gram-negative bacteria (Colangelo et al., 2002; Ferrera et al., 2014), and (ii) the presence of bacteria,
LPS or endotoxin-mediated inflammation strongly contributes to amyloid neurotoxicity (Hammer et al., 2008; Dasari et al., 2011; Blanco et al., 2012; Zhou et al., 2012; Serpente et al., 2014).

\section{CONGO RED STAINING TO IDENTIFY AMYLOID DEPOSITS}

Congo red $\left[3,3^{\prime}-\left(\left[1,1^{\prime}\right.\right.\right.$-biphenyl $]-4,4^{\prime}-$ diyl)bis(4-aminonaphthalene-1-sulfonicacid-disodium salt)], a toxic, water soluble secondary diazo dye, was first synthesized in 1883 and used as a textile dye (Steensma, 2001; Linke, 2006). Due to its toxicity, Congo red's use in textile dying was discontinued, but because of its important spectroscopic properties gained wide use in investigative microbiology (Linke, 2006). The proposed Congo red-mediated staining mechanism suggests a substrate-mediated hydrophobic pi-pi orbital stacking interaction between the aromatic rings of the dye molecules and $\beta$-pleated sheet structures of both amyloids and textiles (Buxbaum and Linke, 2012). Congo red was classically used in microbiological epidemiology as a bacterial-stain, for example, to rapidly identify the presence of virulent forms of gram-negative Shigella where the dye binds the bacterium's unique LPS surface structures (Linke, 2006; Buxbaum and Linke, 2012). Congo red's apple-green birefringent fluorescence under polarized light when bound to amyloid fibrils is currently used as a sensitive diagnostic tool for amyloidosis including the $\mathrm{A} \beta 42$ enriched SPs of AD (Steensma, 2001; Linke, 2006; Buxbaum and Linke, 2012). Interestingly it has very recently been found that (i) LPS is capable of inducing a pathogenic Congo red-sensitive $\beta$-pleated sheet conformation in prion amyloids (Saleem et al., 2014); and (ii) the infectious microbial burden is significantly associated with both $\mathrm{AD}$ development and the propensity of $\mathrm{AD}$ amyloids to be stained by Congo red ( $\mathrm{Bu}$ et al., 2014).

\section{MOLECULAR MIMICRY BETWEEN MITOCHONDRIA AND BACTERIA}

Because mitochondria appear to have originated from bacteria via endosymbiotic relationships that formed very early in the evolutionary history of eukaryotes, cross-reactivity of mitochondria and 
immunological responses to bacterial amyloid or LPS may have deleterious effects on mitochondrial function. This "molecular mimicry" is partially evidenced by extragastric diseases such as the basal ganglia disorder Sydenham's chorea, rheumatic fever, low-grade systemic-inflammatory states and the link to the Firmicute Streptococcus and/or the gram-negative microaerophilic Proteobacteria Helicobacter pylori (Douglas-Escobar et al., 2013; Hayashi, 2013; Hornig, 2013; Roubaud Baudron et al., 2013; Hill et al., 2014). Previous bacterial infection resulting in antibody formation to amyloids or bacterial endotoxins may predispose CNS mitochondria or amyloids to subsequent attack by antibodies resulting in the up-regulation of inflammatory signaling (Bu et al., 2014).

\section{ACTIVATION OF TLR2 BY AMYLOIDS}

TLRs are type I membrane-spanning protein receptors expressed in microglial cells. TLRs play key roles in host protection from microbial-invasion via the activation of the innate-immune system by sensing structurally conserved PAMPs from microbes that are distinguishable from, and not innate to, the host organism (Tükel et al., 2009; Harry, 2013; $\mathrm{Yu}$ and Ye, 2014). Of the 13 currently identified TLRs (TLR1 to TLR13) the microglial TLR2s are activated by amyloid, lipoproteins and other microbial triggers that subsequently induce cytokine production, inflammation, phagocytosis and innate immune defense responses that directly impact CNS homoeostasis and drive neuropathology. More specifically the TLR2/TLR1 complex can recognize biofilm-associated amyloids produced by Firmicutes, Bacteroidetes, and Proteobacteria (Nishimori et al., 2012; Bhattacharjee and Lukiw, 2013; Asti and Gioglio, 2014). Remarkably, the $\mathrm{A} \beta 42$ peptides of $\mathrm{AD}$ that are associated with robust microglia-mediated inflammatory responses also activate TLR2 (Gustot et al., 2013; Yu and Ye, 2014). Of further interest is (i) that microbial amyloids induce pro-inflammatory interleukin IL-17A, a driver of NF-kB signaling and cyclooxygenase- 2 activation, and other potent mediators of inflammatoryresponses such as IL-22 via direct TLR2 activation (Nishimori et al., 2012); and (ii) that increased levels of IL-17A and IL22 are associated with chronic inflammatory diseases including $\mathrm{AD}$ (Zhang et al., 2013).

\section{CONCLUDING REMARKS}

Diverse microbes of the human microbiome generate functional amyloids. Their ability to bind Congo red has provided useful tools for characterizing both microbial- and CNS-derived amyloids (Zhou et al., 2012; Serpente et al., 2014). The large amount of microbialgenerated GI amyloid implicates high potential systemic exposure to bacterial amyloid, and the bioavailability of amyloid to the CNS increases as humans age (Bhattacharjee and Lukiw, 2013; Marques et al., 2013; Tran and Greenwood-Van Meerveld, 2013; Oakley and Tharakan, 2014). Microbial and CNS amyloids are biologically similar in their structure and immunogenicity and complex mechanistic interrelationships between these amyloids are beginning to emerge. It is remarkable (i) that human microbes that produce amyloids such as CsgA and curli, and the $A \beta 42$ peptides that accumulate in $\mathrm{AD}$, are recognized by the same TLR2/TLR1 immune sensor-receptor system of the 13 different TLR-type receptors available; and (ii) that they direct the same up-regulation of IL-17A- and IL22-mediated pro-inflammatory-signaling (Rapsinski et al., 2013; Zhang et al., 2013; Yu and Ye, 2014). Interestingly, CsgA and A $\beta 42$ peptides do not share common amino-acid sequences, only structural similarity in their PAMPs. Microbes or their secretory or degradation products including their amyloids and LPSs are powerful inflammatory activators and inducers of cytokines and complement proteins, affecting vascular permeability and generating free-radicals that further support amyloidogenesis (Hill et al., 2014; Lin et al., 2014). These pathogenic signaling features are also highly characteristic of $\mathrm{AD}$ neuropathology. A more detailed understanding of human microbial ecosystems and their amyloids should give insight into amyloid-misfolding and their contribution to inflammatorysignaling in health, aging and disease. It will certainly be interesting to see: (i) if any microbial-generated amyloids co-localize with the amyloid-dense SP deposits of $\mathrm{AD}$; (ii) if GI tract microbiome-derived amyloids become more available systemically as humans age; and (iii) what the evolution and nature of amyloid-related communication between the GI-tract and the CNS has on the development or propagation of amyloidogenesis in pro-inflammatory degenerative disease.

\section{ACKNOWLEDGMENTS}

This work was presented in part at the Alzheimer Association International Conference 2013 (AAIC 2013) Annual Meeting held in Boston MA, USA and at the AAIC 2014 held in Copenhagen, Denmark. Sincere thanks are extended to Drs. PN Alexandrov, F Culicchia, C Eicken and $C$ Hebel for short post-mortem interval (PMI) human brain tissues or extracts, miRNA array work and initial data interpretation, and to AI Pogue, D Guillot and J Lockwood for expert technical assistance. Additional thanks are extended to the many physicians and neuropathologists of Canada and the USA who have provided high quality, short post-mortem interval human brain and retinal tissues for study. Additional human control and AD brain tissues were provided by the Memory Impairments and Neurological Disorders (MIND) Institute and the University of California, Irvine Alzheimer's Disease Research Center (UCI-ADRC; NIA P50 AG16573). Research on miRNA in the Lukiw laboratory involving the innateimmune response in $\mathrm{AD}$, amyloidogenesis and neuro-inflammation was supported through a COBRE III Pilot Project NIH/NIGMS Grant P30-GM103340, an unrestricted grant to the LSU Eye Center from Research to Prevent Blindness (RPB); the Louisiana Biotechnology Research Network (LBRN) and NIH grants NEI EY006311, NIA AG18031 and NIA AG038834.

\section{REFERENCES}

Alexandrov, P. N., Pogue, A., Bhattacharjee, S., and Lukiw, W. J. (2011). Retinal amyloid peptides and complement factor $\mathrm{H}$ in transgenic models of Alzheimer's disease. Neuroreport 22, 623-627. doi: 10.1097/WNR.0b013e3283497334

Alonso, R., Pisa, D., Marina, A. I., Morato, E., Rábano, A., and Carrasco, L. (2014b). Fungal infection in patients with Alzheimer's disease. J. Alzheimers Dis. 41, 301-311. doi: 10.3233/JAD-132681

Alonso, R., Pisa, D., Rábano, A., and Carrasco, L. (2014a). Alzheimer's disease and disseminated mycoses. Eur. J. Clin. Microbiol. Infect. 
Dis. 33, 1125-1132. doi: 10.1007/s10096-0132045-Z

Armstrong, R. A. (2006). Plaques and tangles and the pathogenesis of Alzheimer's disease. Folia Neuropathol. 44, 1-11.

Asti, A., and Gioglio, L. (2014). Can a bacterial endotoxin be a key factor in the kinetics of amyloid fibril formation? J. Alzheimers. Dis. 39, 169-179. doi: 10.3233/JAD-131394

Badtke, M. P., Hammer, N. D., and Chapman, M. R. (2009). Functional amyloids signal their arrival. Sci. Signal. 2:pe43. doi: 10.1126/scisignal. 280pe43

Bhattacharjee, S., and Lukiw, W. J. (2013). Alzheimer's disease and the microbiome. Front. Cell. Neurosci. 7:153. doi: $10.3389 /$ fncel.2013.00153

Blanco, L. P., Evans, M. L., Smith, D. R., Badtke, M. P., and Chapman, M. R. (2012). Diversity, biogenesis and function of microbial amyloids. Trends Microbiol. 20, 66-73. doi: 10.1016/j.tim.2011.11.005

Boutajangout, A., and Wisniewski, T. (2013). The innate immune system in Alzheimer's disease. Int. J. Cell Biol. 2013, 576383. doi: 10.1155/2013/576383

Bu, X. L., Yao, X. Q., Jiao, S. S., Zeng, F., Liu, Y. H., Xiang, Y., et al. (2014). A study on the association between infectious burden and Alzheimer's disease. Eur. J. Neurol. doi: 10.1111/ene.12477. [Epub ahead of print].

Buxbaum, J. N., and Linke, R. P. (2012). A molecular history of the amyloidoses. J. Mol. Biol. 421, 142-159. doi: 10.1016/j.jmb.2012.01.024

Colangelo, V., Schurr, J., Ball, M. J., Pelaez, R. P., Bazan, N. G., and Lukiw, W. J. (2002). Gene expression profiling of 12633 genes in Alzheimer hippocampal CA1: transcription and neurotrophic factor down-regulation and upregulation of apoptotic and pro-inflammatory signaling. J. Neurosci. Res. 70, 462-473. doi: 10.1002/jnr.10351

Cui, J. G., Hill, J. M., Zhao, Y., and Lukiw, W. J. (2007). Expression of inflammatory genes in the primary visual cortex of late-stage Alzheimer's disease. Neuroreport 18, 115-119. doi: 10.1097/WNR.0b013e32801198bc

Dasari, M., Espargaro, A., Sabate, R., Lopez del Amo, J. M., Fink, U., Grelle, G., et al. (2011). Bacterial inclusion bodies of Alzheimer's disease $\beta$-amyloid peptides can be employed to study native-like aggregation intermediate states. Chembiochem 12, 407-423. doi: 10.1002/cbic.201000602

Douglas-Escobar, M., Elliott, E., and Neu, J. (2013). Effect of intestinal microbial ecology on the developing brain. JAMA Pediatr. 167, 374-379. doi: 10.1001/jamapediatrics.2013.497

Ferrera, D., Mazzaro, N., Canale, C., and Gasparini, L. (2014). Resting microglia react to $\mathrm{A} \beta 42$ fibrils but do not detect oligomers or oligomer-induced neuronal damage. Neurobiol. Aging. 35, 2444-2457. doi: 10.1016/j.neurobiolaging.2014.05.023

Furukawa, Y., and Nukina, N. (2013). Functional diversity of protein fibrillar aggregates from physiology to RNA granules to neurodegenerative diseases. Biochim. Biophys. Acta. 1832, 1271-1278. doi: 10.1016/j.bbadis.2013.04.011

Goldschmidt, L., Teng, P. K., Riek, R., and Eisenberg, D. (2010). Identifying the amylome, proteins capable of forming amyloid-like fibrils. Proc.
Natl. Acad. Sci. U.S.A. 107, 3487-3492. doi: 10.1073/pnas.0915166107

Gustot, A., Raussens, V., Dehousse, M., Dumoulin, M., Bryant, C. E., Ruysschaert, J. M., et al. (2013). Activation of innate immunity by lysozyme fibrils is critically dependent on cross- $\beta$ sheet structure. Cell. Mol. Life Sci. 70, 2999-3012. doi: 10.1007/s00018-012-1245-5

Hammer, N. D., Wang, X., McGuffie, B. A., and Chapman, M. R. (2008). Amyloids: friend or foe? J. Alzheimers Dis. 13, 407-419.

Harry, G. J. (2013). Microglia during development and aging. Pharmacol. Ther. 139, 313-326 doi: 10.1016/j.pharmthera.2013.04.013

Hattori, M., and Taylor, T. D. (2009). The human intestinal microbiome: a new frontier of human biology. DNA Res. 16, 1-12. doi: 10.1093/dnares/dsn033

Hayashi, M. (2013). [Anti-basal ganglia antibody]. Brain Nerve 65, 377-384.

Hill, J. M., Clement, C., Pogue, A. I., Bhattacharjee, S., Zhao, Y., and Lukiw, W. J. (2014). Pathogenic microbes, the microbiome, and Alzheimer's disease (AD). Front. Aging Neurosci. 6:127. doi: 10.3389/fnagi.2014.00127

Hornig, M. (2013). The role of microbes and autoimmunity in the pathogenesis of neuropsychiatric illness. Curr. Opin. Rheumatol. 25, 488-795. doi: 10.1097/BOR.0b013e3283 6208 de

Jones, B. M., Bhattacharjee, S., Dua, P., Hill, J. M., Zhao, Y., and Lukiw, W. J. (2014). Regulating amyloidogenesis through the natural triggering receptor expressed in myeloid/microglialcells 2 (TREM2). Front. Cell. Neurosci. 8:94. doi: 10.3389/fncel.2014.00094

Lin, C. S., Chang, C. J., Lu, C. C., Martel, J., Ojcius, D. M., Ko, Y. F., et al. (2014). Impact of the gut microbiota, prebiotics, and probiotics on human health and disease. Biomed. J. 37, 259-268. doi: 10.4103/2319-4170.138314

Linke, R. P. (2006). "Congo red staining of amyloid; improvements and practical guide for a more precise diagnosis of amyloid and the different amyloidoses. Chapter 11.1," in Protein Misfolding, Aggregation, and Conformational Diseases Part A: Protein Aggregation and Conformational Diseases, eds V. N. Uversky and A. L. Fink (New York, NY: Springer), 239-276. doi: 10.1007/0-387-259198_12

Lukiw, W. J. (2012). Amyloid beta (A $\beta$ ) peptide modulators and other current treatment strategies for Alzheimer's disease (AD). Expert Opin. Emerg. Drugs 17, 43-60. doi: 10.1517/14728214.2012.672559

Marques, F., Sousa, J. C., Sousa, N., and Palha, J. A. (2013). Blood-brain-barriers in aging and in Alzheimer's disease. Mol. Neurodegener. 8, 38. doi: 10.1186/1750-1326-8-38

Nishimori, J. H., Newman, T. N., Oppong, G. O., Rapsinski, G. J., Yen, J. H., Biesecker, S. G., et al. (2012). Microbial amyloids induce interleukin 17A (IL-17A) and IL-22 responses via Tolllike receptor 2 (TLR2) activation in the intestinal mucosa. Infect. Immun. 80, 4398-4408. doi: 10.1128/IAI.00911-12

Oakley, R., and Tharakan, B. (2014). Vascular hyperpermeability and aging. Aging Dis. 5, 114-125. doi: 10.14336/AD.2014.0500114
O'Brien, R. J., and Wong, P. C. (2011). Amyloid precursor protein processing and Alzheimer's disease. Annu. Rev. Neurosci. 34, 185-204. doi: 10.1146/annurev-neuro-061010-113613

Rapsinski, G. J., Newman, T. N., Oppong, G. O., van Putten, J. P., and Tükel, Ç. (2013). CD14 protein acts as an adaptor molecule for the immune recognition of Salmonella curli fibers. J. Biol. Chem. 288, 14178-14188. doi: 10.1074/jbc.M112. 447060

Roubaud Baudron, C., Franceschi, F., Salles, N., and Gasbarrini, A. (2013). Extragastric diseases and Helicobacter pylori. Helicobacter. 18(Suppl. 1), 44-51. doi: 10.1111/hel.12077

Saleem, F., Bjorndahl, T. C., Ladner, C. L., PerezPineiro, R., Ametaj, B. N., and Wishart, D. S. (2014). Lipopolysaccharide induced conversion of recombinant prion protein. Prion $8,221-232$. doi: 10.4161/pri.28939

Schwartz, K., and Boles, B. R. (2013). Microbial amyloids - functions and interactions within the host. Curr. Opin. Microbiol. 16, 93-99. doi: 10.1016/j.mib.2012.12.001

Serpente, M., Bonsi, R., Scarpini, E., and Galimberti, D. (2014). Innate immune system and inflammation in Alzheimer's disease: from pathogenesis to treatment. Neuroimmunomodulation 21, 79-87. doi: 10.1159/000356529

Steensma, D. P. (2001). "Congo" red: out of Africa? Arch. Pathol. Lab. Med. 125, 250-252.

Syed, A. K., and Boles, B. R. (2014). Fold modulating function: bacterial toxins to functional amyloids. Front. Microbiol. 5:401. doi: 10.3389/fmicb.2014.00401

Takeda, S., Sato, N., and Morishita, R. (2014). Systemic inflammation, blood-brain barrier vulnerability and cognitive/non-cognitive symptoms in Alzheimer disease: relevance to pathogenesis and therapy. Front. Aging Neurosci. 6:171. doi: 10.3389/fnagi.2014.00171

Teng, P. K., Anderson, N. J., Goldschmidt, L., Sawaya, M. R., Sambashivan, S., and Eisenberg, D. (2012). Ribonuclease A suggests how proteins selfchaperone against amyloid fiber formation. Protein Sci. 21, 26-37. doi: 10.1002/pro.754

Tran, L., and Greenwood-Van Meerveld, B. (2013). Age-associated remodeling of the intestinal epithelial barrier. J. Gerontol. ABiol. Sci. Med. Sci. 68, 1045-1056. doi: 10.1093/gerona/ glt106

Tükel, C., Wilson, R. P., Nishimori, J. H., Pezeshki, M. Chromy, B. A., and Bäumler, A. J. (2009). Responses to amyloids of microbial and host origin are mediated through toll-like receptor 2. Cell Host Microbe. 6, 45-53. doi: 10.1016/j.chom.2009.05.020

Van Broeck, B., Van Broeckhoven, C., and KumarSingh, S. (2007). Current insights into molecular mechanisms of Alzheimer disease and their implications for therapeutic approaches. Neurodegener. Dis. 4, 349-365. doi: 10.1159/000 105156

Yu, Y., and Ye, R. D. (2014). Microglial A $\beta$ receptors in Alzheimer's disease. Cell. Mol. Neurobiol. 35, 71-83. doi: 10.1007/s10571-014-0101-6

Zhang, H., Ma, Q., Zhang, Y. W., and Xu, H. (2012). Proteolytic processing of Alzheimer's $\beta$-amyloid precursor protein. J. Neurochem. 120, 9-21. doi: 10.1111/j.1471-4159.2011.07519.x 
Zhang, J., Ke, K. F., Liu, Z., Qiu, Y. H., and Peng, Y. P. (2013). Th17 cell-mediated neuroinflammation is involved in neurodegeneration of A 342 -induced Alzheimer's disease model rats. PLoS ONE 8:e75786. doi: 10.1371/journal.pone. 0075786

Zhao, Y., Bhattacharjee, S., Jones, B. M., Dua, P., Alexandrov, P. N., Hill, J. M., et al. (2013). Regulation of TREM2 expression by an NF- $\mathrm{kB}$-sensitive miRNA-34a. Neuroreport 24, 318-323. doi: 10.1097/WNR.0b013e328 $35 \mathrm{fb} 6 \mathrm{~b} 0$

Zhou, Y., Blanco, L. P., Smith, D. R., and Chapman, M. R. (2012). Bacterial amyloids. Methods Mol. Biol. 849, 303-320. doi: 10.1007/978-1-61779-551-0_21
Zhou, Z. D., Chan, C. H., Ma, Q. H., Xu, X. H., Xiao, Z. C., and Tan, E. K. (2011). The roles of amyloid precursor protein in neurogenesis: implications to pathogenesis and therapy of Alzheimer disease. Cell Adh. Migr. 5, 280-292. doi: 10.4161/cam.5.4.16986

Conflict of Interest Statement: The authors declare that the research was conducted in the absence of any commercial or financial relationships that could be construed as a potential conflict of interest.

Received: 10 September 2014; paper pending published: 26 November 2014; accepted: 21 January 2015; published online: 10 February 2015.
Citation: Hill JM and Lukiw WJ (2015) Microbialgenerated amyloids and Alzheimer's disease (AD). Front. Aging Neurosci. 7:9. doi: 10.3389/fnagi. 2015.00009

This article was submitted to the journal Frontiers in Aging Neuroscience.

Copyright (c) 2015 Hill and Lukiw. This is an openaccess article distributed under the terms of the Creative Commons Attribution License (CC BY). The use, distribution or reproduction in other forums is permitted, provided the original author(s) or licensor are credited and that the original publication in this journal is cited, in accordance with accepted academic practice. No use, distribution or reproduction is permitted which does not comply with these terms. 